\title{
A Crash Surrogate Metric considering Traffic Flow Dynamics in a Motorway Corridor
}

\author{
Xu Wang' and Kai Liu $\mathbb{D}^{2}$ \\ ${ }^{1}$ Griffith School of Engineering, Griffith University, Gold Coast, QLD 4222, Australia \\ ${ }^{2}$ School of Transportation and Logistics, Dalian University of Technology, Dalian 116024, China \\ Correspondence should be addressed to Kai Liu; liukai@dlut.edu.cn
}

Received 20 February 2018; Revised 22 April 2018; Accepted 6 May 2018; Published 27 June 2018

Academic Editor: Zhiyuan Liu

Copyright (c) $2018 \mathrm{Xu}$ Wang and Kai Liu. This is an open access article distributed under the Creative Commons Attribution License, which permits unrestricted use, distribution, and reproduction in any medium, provided the original work is properly cited.

\begin{abstract}
We proposed a new crash surrogate metric, i.e., the maximum disturbance that a car following scenario can accommodate, to represent potential crash risks with a simple closed form. The metric is developed in consideration of traffic flow dynamics. Then, we compared its performance in predicting the rear-end crash risks for motorway on-ramps with other two surrogate measures (time to collision and aggregated crash index). To this end, a one-lane on-ramp of Pacific Motorway, Australia, was selected for this case study. Due to the lack of crash data on the study site, historical crash counts were merged according to levels of service (LOS) and then converted into crash rates. In this study, we used the societal risk index to represent the crash surrogate indicators and built relationships with crash rates. The final results show that (1) the proposed metric and aggregated crash index are superior to the time to collision in predicting the rear-end crash risks for on-ramps; (2) they have a relatively similar performance, but due to the simple calculation, the proposed metric is more applicable to some real-world cases compared with the aggregated crash index.
\end{abstract}

\section{Introduction}

According to the World Health Organization [1], approximately 1.24 million people died and over 50 million were injured in road crashes all over the world. More importantly, road crashes have been the leading cause of death for young people aged 15-29 years. In Australia, the social cost of road crashes has been estimated as a devastating AU $\$ 27$ billion per annum [2]. It has been well recognized that freeway crashes are much more dangerous than those on urban streets due to their severity [3-5]. According to United States Department of Transportation [6], the proportion of fatal crashes on freeways is significantly higher than that on other road types. Further, on-ramp areas of freeways may be prone to relatively more traffic crashes than other sections of freeways (Cheng et al., 2017) [7] because drivers have been facing a high competition for merging space in such areas $[8,9]$. In this regard, capable solutions need to be sought in order to proactively assess or predict on-ramp crash.

Considerable research efforts have been carried out over the past fifty years on developing count-data regression models to predict crash frequency [10] and to investigate crash casualty of different collision types [11, 12], most of which are purely dependent on statistical techniques. These count-data regression models, in accordance with distinct statistical assumptions, represent the relationship between number of crashes and its contributing factors which have little, if not none, consideration of traffic flow dynamics $[13,14]$. Since the 1970 s, some researchers began to use crash surrogate measure to evaluate road safety (e.g., [1519]). Only recently has a consensus emerged concerning the definition of a crash surrogate, which is defined based on the relationship [20,21]: the number of crashes expected to occur on an entity during a certain period of time $(\lambda)=$ the number of crash surrogates occurring on an entity in that time $(\pi) *$ crash-to-surrogate ratio for that entity $(c)$, mathematically,

$$
\lambda=\pi \cdot c \text {. }
$$

A few crash surrogate metrics have been proposed and designed, including the time to collision (TTC), the deceleration rate to avoid crash (DRAC), and the crash potential index (CPI). However, as proposed by Kuang et al. [22], these models are incapable of representing crash surrogates on freeways, especially saturated freeways where 
a minor disturbance can result in a rear-end crash due to very high speeds and small headways. For example, if the time headway between two consecutive vehicles on a freeway is 0.5 seconds and their speeds are equal at $110 \mathrm{~km} / \mathrm{hr}$, all these crash surrogate metrics will identify it as a safe scenario. In other words, the abovementioned crash surrogate metrics fail to assess crash risks of this particular car following scenario, which may lead to erroneous judgement. In this regard, Kuang et al. [22] proposed a tree-structured crash surrogate metric by imposing a hypothetical disturbance to the leading vehicle. An aggregated crash index (ACI) was proposed to combine eight possible scenarios caused by the imposed hypothetical disturbance. According to the validation, the ACI outperforms the traditional TTC based surrogate metrics in representing freeway rear-end crash risks.

The biggest disadvantage of this tree-structured crash surrogate measure is that a closed form is not available due to this rather complicated tree structure. It, unfortunately, limits the metric's applicability to deal with real-world problems. For example, the model is naturally applicable to optimize the traffic operations of connected and automated vehicles in order to achieve the highest safety level. Unfortunately, as there is no closed form for ACI, the traditional optimization models cannot be used and only simulation based optimization models can be considered as an alternative. As such, it is difficult to analyze the analytical properties of the optimization results.

In this paper, we proposed a new concept of traffic state vulnerability to develop a simplified crash surrogate metric (SCSM). Traffic state vulnerability is defined as the maximum disturbance that a car following scenario can accommodate. With this new concept, the SCSM with a closed form was proposed. We further compared the performances of this new surrogate metric, ACI, and the conventional TTC based surrogate metrics for on-ramps. According to a comparative analysis, although the SCSM has a much simpler form, it has more or less similar performance compared to ACI, which outperforms the TTC. The rest of the paper is organized as follows. Section 2 briefs the TTC and ACI. The SCSM is introduced in Section 3. A comparative analysis is carried out in Section 4. Section 5 concludes this study and points out some future research directions and possible application of the new metric.

\section{Literature Review}

Multiple researchers have made great efforts to proactively predict accident risks so that various surrogate measures of safety have been designed and developed over past decades. Among them, the most widely used surrogate measure of TTC is defined as the time remaining until a collision will occur between two vehicles if the collision course and speed difference are maintained [23], or mathematically,

$$
\text { TTC }= \begin{cases}\frac{D_{l-f}}{v_{f}-v_{l}}, & \text { if } v_{f}>v_{l} \\ \infty, & \text { otherwise }\end{cases}
$$

where $v_{f}$ is the speed of follower's vehicle in $\mathrm{m} / \mathrm{s}, v_{l}$ is the speed of leader's vehicle in $\mathrm{m} / \mathrm{s}$, and $D_{l-f}$ is the gap between the two vehicles in metre. All TTC values need to compare with its threshold which has ranged from $1.5 \mathrm{~s}$ to $4 \mathrm{~s}$ in previous studies [24]. Only those car following scenarios with TTC values less than its threshold could be judged as risky.

The defects of the TTC contributed to the advance of other safety surrogate measures [25-27]. However, there are some shortcomings present in these methods. Firstly, boundary condition is improperly obeyed. For example, scenarios in which the speed of leading vehicle is higher than that of following vehicle are identified as safe situations. However, this condition boundary is not applicable to freeways with saturated traffic flow, as vehicles travelling at high speeds have relatively small differences in speeds. Accordingly, some potential risks on freeways may not be accurately measured. Secondly, the motorists' reaction time, as an essential parameter in crash risk assessment, fails to be considered in these surrogate metrics. Thirdly, the setting of threshold, like the TTC, lacks a specific judgement criterion. To address the drawbacks, Kuang et al. [22] proposed a safety surrogate measure called ACI which can better assess conflicts occurring on saturated freeways. Built on a probabilistic causal model, they first developed a tree-structured crash model being able to impose hypothetical disturbance. Eight possible conflict types under the model were recognized through four stepped condition levels.

The advantages of the ACI lie in two aspects. First, it was proposed for the risk assessment of car following scenarios on saturated freeways. Accordingly, it is able to eliminate false negative errors (in the saturated traffic flow, the scenarios where the speed of following vehicle is less than that of leading vehicle, but the speed difference is slight, are mistakenly viewed as safe). Second, the ACI incorporates two essential parameters like driver's reaction time and maximum available deceleration rate (MADR) in crash mechanism. They are invariably considered in the form of distribution instead of a fixed value.

However, its complex probability calculation limits its applicability to assess potential risks in the real world. As a result, the Monte Carlo method (MCM) may be the only method to simplify the complicated calculation processes involved in the ACI.

\section{A Simplified Crash Surrogate Metric}

Based on the notion of traffic state vulnerability, a new simplified crash surrogate metric (SCSM), with a closed form, is proposed. It is defined as the maximum disturbance that a traffic state could accommodate, namely, the ultimate capacity that a car following scenario can avoid a collision. It can be categorised into two scenarios based on the occurrence probability of a crash.

Scenario I. A crash will not occur under the condition that

$$
v_{f} \leq v_{l}-\delta
$$


That is,

$$
\delta \leq v_{l}-v_{f},
$$

where $\delta$ is the maximum interference that a traffic state can withstand.

Scenario II. A crash is possible to occur under the condition that

$$
v_{f}>v_{l}-\delta
$$

That is,

$$
\delta>v_{l}-v_{f}
$$

Apparently, as $v_{f}$ is greater than $v_{l}-\delta$, a crash will occur if both vehicles maintain the same speed. In other words, the modified time to collision with respect to disturbance $\delta$ can be calculated as

$$
\mathrm{TTC}_{\delta}=\frac{d_{l-f}}{v_{f}-\left(v_{l}-\delta\right)} .
$$

To further evaluate the crash risks of a car following state, we need to compare the predicted remaindering time to crash and the threshold of time to collision. If (8) holds, the following vehicle is able to timely decelerate and a crash could be avoided. Otherwise, a crash will occur. In this research, according to the previous researchers' experience [24, 28, 29], $3 \mathrm{~s}$ as the critical value can be suitable to assess crash risks on freeways with saturated flow.

$$
\frac{d_{l-f}}{v_{f}-\left(v_{l}-\delta\right)} \geq \tau .
$$

$d_{l-f}$ can be estimated by

$$
d_{l-f}=v_{f} h-L_{l},
$$

where $h$ is the time headway of this car following scenario and $L_{l}$ is the length of the leading vehicle. By substituting (9) into (8), we have

$$
\frac{v_{f} h-L_{l}}{v_{f}-\left(v_{l}-\delta\right)} \geq \tau .
$$

As $v_{f}-\left(v_{l}-\delta\right)>0,(10)$ is equivalent to

$$
\left(v_{l}-v_{f}\right)<\delta \leq\left(v_{l}-v_{f}\right)+\frac{v_{f} h-L_{l}}{\tau} .
$$

In other words, as long as a disturbance satisfies (11), a crash is avoidable; otherwise, a crash will occur. By combining the conditions for Scenarios I and II, we can conclude that a crash will not occur if

$$
0 \leq \delta \leq\left(v_{l}-v_{f}\right)+\frac{v_{f} h-L_{l}}{\tau} .
$$

\begin{tabular}{|c|c|c|}
\hline Time period & LOS & Crash counts \\
\hline 00:00-01:00 & $A \& B$ & 0 \\
\hline 01:00-02:00 & $A \& B$ & 1 \\
\hline 02:00-03:00 & $A \& B$ & 1 \\
\hline 03:00-04:00 & $A \& B$ & 0 \\
\hline 04:00-05:00 & $A \& B$ & 0 \\
\hline 05:00-06:00 & $\mathrm{D}$ & 1 \\
\hline $06: 00-07: 00$ & $\mathrm{E}$ & 2 \\
\hline 07:00-08:00 & $\mathrm{E}$ & 3 \\
\hline 08:00-09:00 & $\mathrm{D}$ & 1 \\
\hline 09:00-10:00 & $\mathrm{D}$ & 1 \\
\hline 10:00-11:00 & $\mathrm{C}$ & 0 \\
\hline 11:00-12:00 & $\mathrm{C}$ & 1 \\
\hline $12: 00-13: 00$ & $\mathrm{C}$ & 1 \\
\hline 13:00-14:00 & $\mathrm{C}$ & 0 \\
\hline 14:00-15:00 & $\mathrm{C}$ & 2 \\
\hline $15: 00-16: 00$ & $\mathrm{D}$ & 2 \\
\hline $16: 00-17: 00$ & $\mathrm{D}$ & 2 \\
\hline $17: 00-18: 00$ & $\mathrm{E}$ & 3 \\
\hline 18:00-19:00 & $\mathrm{D}$ & 1 \\
\hline 19:00-20:00 & $\mathrm{C}$ & 1 \\
\hline $20: 00-21: 00$ & $\mathrm{C}$ & 0 \\
\hline $21: 00-22: 00$ & A \& B & 1 \\
\hline $22: 00-23: 00$ & $A \& B$ & 0 \\
\hline $23: 00-00: 00$ & $A \& B$ & 0 \\
\hline
\end{tabular}

Namely, $\left(v_{l}-v_{f}\right)+\left(v_{f} h-L_{l}\right) / \tau$ is the maximum disturbance that a car following scenario is able to accommodate. The greater $\delta$ is, the larger disturbance a traffic state can accommodate; namely, a car following scenario has better capacity to resist on-ramp rear-end crash risks.
TABLE 1: 24-hour based crash counts on the study on-ramp from year 2005 to year 2013.

\section{A Comparative Analysis}

4.1. Crash Data Processing. In this study, crash data were provided by Department of Transport and Main Roads (DTMR) and compiled based on annual daily hourly crash counts. By means of accident coordinates, all rear-end crashes occurring on the study on-ramp from year 2005 to year 2013 were extracted and listed in Table 1. Due to the lack of crash data, we determined to merge current crash counts based on levels of service (LOS). For this purpose, each time period in the 24 hours was marked with LOS based on the hourly traffic flow rates provided by DTMR from 15 to 19 April 2013 (5 workdays). Four groups of LOS were considered in this study: A\&B, C, D, and E. According to HCM 2010, the determination of LOS highly depends on traffic volumes and capacity. Accordingly, LOS acted as an important role in this research. Based on the processed data, crash rates under the corresponding LOS from year 2005 to year 2013 can be calculated and presented in Table 2 .

4.2. Simulation Model Development and Validation. In this research, VISSIM was used to simulate and reproduce downto-earth traffic scenarios of the research segment [30]. An onramp of northbound Pacific Motorway, Queensland, adjacent to the largest shopping centre of suburb Nerang, was chosen as the research site, as it involves slight traffic delay and relatively many weaving manoeuvres during rush hours and 


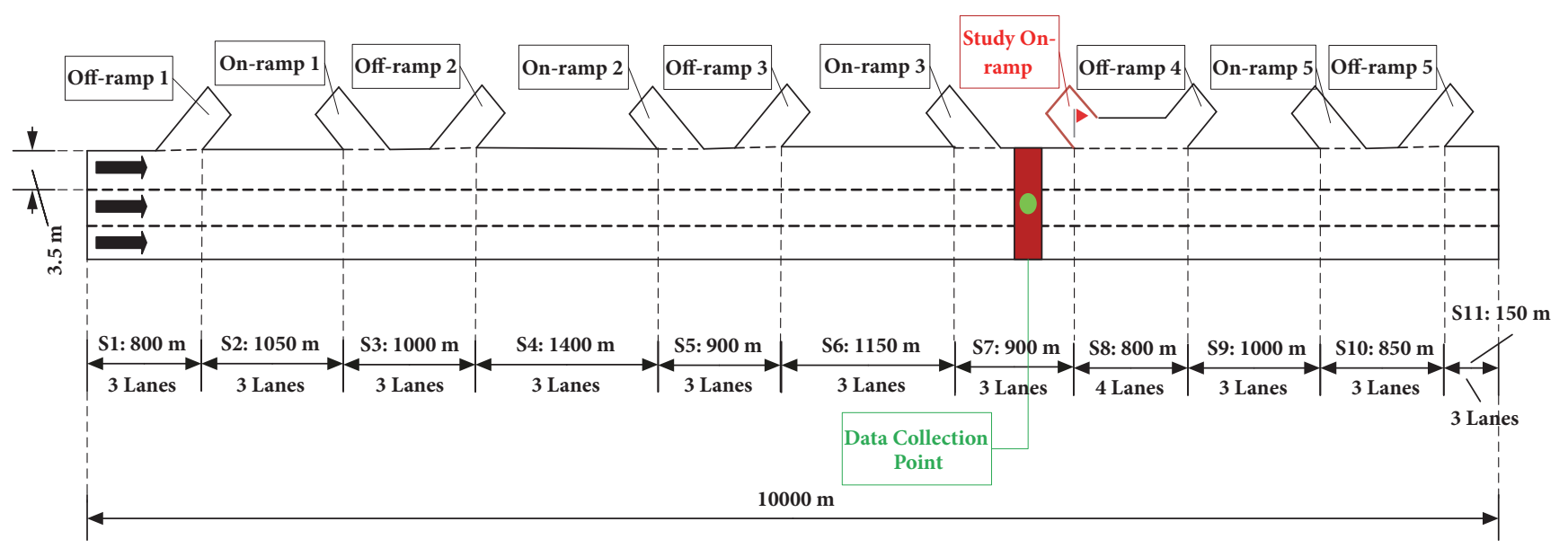

Figure 1: The sketch map of research area.

TABLE 2: Crash rate in the past nine years under the corresponding LOS.

\begin{tabular}{lccc}
\hline LOS & $\begin{array}{c}\text { The number of } \\
\text { time periods }\end{array}$ & Crash counts & $\begin{array}{c}\text { Crash rate } \\
\text { (counts/hr) }\end{array}$ \\
\hline A\&B & 8 & 3 & 0.38 \\
C & 7 & 5 & 0.71 \\
D & 6 & 8 & 1.33 \\
E & 3 & 8 & 2.67 \\
\hline
\end{tabular}

has always been regarded as a bottleneck by Gold Coast City Council (GCCC). There were 36 data collection points set up on the 360 -metre on-ramp with a spacing of 10 metres. To reproduce the impact of the up- and down-stream traffic flow on the research on-ramp, the simulation model was extended to a length of 10 kilometres. Additional 4 on-ramps and 5 offramps were involved within 10 kilometres. The sketch map of the research area is shown in Figure 1. There was no accident occurring during the data collection process and no major modification of the research on-ramp and its circumambient ramps from year 2005 to year 2013. All settings associated with geometrical characteristics need to be coded based on Google Earth Pro and behavioural parameters in the Wiedemann 99 model need to be calibrated with the real situations. According to the existing data, the average traffic flow rates under four different LOS can be derived through loop detectors setup at upstream 200 metres of off-ramp 1, which was used as traffic volume input in VISSIM. Similarly, the average number of merging traffic and diverging traffic for each on-ramp and off-ramp can also be obtained and shown in Table 3. Take traffic scenario 1 as an example. The traffic volume input for on-ramp 1,2,3, and 5 and the study on-ramp account for $7.1 \%, 6.5 \%, 4.6 \%, 4.1 \%$, and $7.5 \%$ of their corresponding mainline traffic, respectively. Similarly, there are $5.4 \%, 5.3 \%, 7.2 \%, 6.3 \%$, and $6.8 \%$ of mainline traffic diverging into off-ramp $1,2,3,4$, and 5 .

To validate the effectiveness of VISSIM in generating dynamic vehicle behaviour, a bottleneck close to the study onramp in the motorway corridor was identified and used for video recording. Four videos need to be recorded and to meet the following criterion: traffic volumes in the four videos are as close as possible to those generated by simulation models at the same location. Then, the traffic trajectories for 20 random consecutive vehicles in each video were extracted and compared with those generated from simulation models. Four error tests were herein carried out to evaluate the differences between the simulated results and the observed data: (1) root mean square error (RMSE), (2) root mean square percentage error (RMSPE), (3) mean percentage error (MPE), and (4) Theil's inequality coefficient $(U)$, mathematically,

$$
\begin{aligned}
& \mathrm{RMSE}=\sqrt{\frac{1}{N_{0}} \sum_{n=1}^{N_{0}}\left(y_{n}^{s}-y_{n}^{0}\right)^{2}} \\
& \mathrm{RMSPE}=\sqrt{\frac{1}{N_{0}} \sum_{n=1}^{N_{0}}\left(\frac{y_{n}^{s}-y_{n}^{0}}{y_{n}^{0}}\right)^{2}} \\
& \mathrm{MPE}=\frac{1}{N_{0}} \sum_{n=1}^{N_{0}}\left(\frac{y_{n}^{s}-y_{n}^{0}}{y_{n}^{0}}\right) \\
& U=\frac{\sqrt{\left(1 / N_{0}\right) \sum_{n=1}^{N_{0}}\left(y_{n}^{s}-y_{n}^{0}\right)^{2}}}{\sqrt{\left(1 / N_{0}\right) \sum_{n=1}^{N_{0}}\left(y_{n}^{s}\right)^{2}}+\sqrt{\left(1 / N_{0}\right) \sum_{n=1}^{N_{0}}\left(y_{n}^{0}\right)^{2}}}
\end{aligned}
$$

where $y_{n}^{s}$ is the simulation value (speed) of the $n$th vehicle, $y_{n}^{0}$ is the field value (speed) of the $n$th vehicle, and $N_{0}$ is the number of vehicles observed or simulated. The error tests of speeds were shown in Table 4. For any error tests, the difference between simulated speeds and observed speeds is the greatest under LOS $A \& B$, as the speeds for vehicles under LOS A\&B are close to free speeds, which is difficult to capture through video recording. Besides, the selection of driving speeds is highly free under LOS A\&B. As a result, a larger difference in speeds is inevitable. For a speed limit of $110 \mathrm{~km} / \mathrm{hr}(30.56 \mathrm{~m} / \mathrm{s})$, an error of $3.37 \mathrm{~m} / \mathrm{s}$ is in the acceptable range. The values for RMSPE are less than $15 \%$ across all groups. The largest value for MPE is positive $7.69 \%$, which 
TABLE 3: The traffic flow configuration for each on-ramp and off-ramp.

\begin{tabular}{|c|c|c|c|c|}
\hline Traffic scenario & 1 & 2 & 3 & 4 \\
\hline LOS & $A \& B$ & $\mathrm{C}$ & $\mathrm{D}$ & $\mathrm{E}$ \\
\hline Freeway input (vehs/hr) & 1830 & 3960 & 5220 & 6360 \\
\hline Off-ramp 1 output (vehs/r) & $99(5.4 \%)$ & $245(6.1 \%)$ & $298(5.7 \%)$ & $426(6.7 \%)$ \\
\hline On-ramp 1 input (vehs/hr) & $123(7.1 \%)$ & $238(6.4 \%)$ & $315(6.4 \%)$ & $362(6.1 \%)$ \\
\hline Off-ramp 2 output (vehs/hr) & $98(5.3 \%)$ & $233(5.9 \%)$ & $346(6.6 \%)$ & $384(6.1 \%)$ \\
\hline On-ramp 2 input (vehs/hr) & $114(6.5 \%)$ & $223(6.0 \%)$ & $303(6.2 \%)$ & $414(7.0 \%)$ \\
\hline Off-ramp 3 output (vehs/hr) & $135(7.2 \%)$ & $334(7.7 \%)$ & $441(8.5 \%)$ & $512(8.1 \%)$ \\
\hline On-ramp 3 input (vehs/hr) & $80(4.6 \%)$ & $189(5.2 \%)$ & $304(6.4 \%)$ & $401(6.9 \%)$ \\
\hline Study on-ramp input (vehs/hr) & $136(7.5 \%)$ & $291(7.6 \%)$ & $405(8.0 \%)$ & $466(7.5 \%)$ \\
\hline Off-ramp 4 output (vehs/hr) & $123(6.3 \%)$ & $276(6.7 \%)$ & $364(5.2 \%)$ & $488(7.3 \%)$ \\
\hline On-ramp 5 input (vehs/hr) & $75(4.1 \%)$ & $165(4.3 \%)$ & $190(3.9 \%)$ & $279(4.5 \%)$ \\
\hline Off-ramp 5 output (vehs/hr) & $130(6.8 \%)$ & $281(7.0 \%)$ & $329(6.5 \%)$ & $466(7.2 \%)$ \\
\hline
\end{tabular}

TABLE 4: Error tests of speeds.

\begin{tabular}{lcccc}
\hline LOS group & RMSE (m/s) & RMSPE (\%) & MPE (\%) & $U(\%)$ \\
\hline A\&B & 3.37 & 14.19 & 7.69 & 0.28 \\
C & 3.28 & 13.73 & 6.67 & 0.26 \\
D & 2.64 & 11.86 & 5.75 & 0.23 \\
E & 2.68 & 11.51 & 5.51 & 0.24 \\
\hline
\end{tabular}

indicates that the simulated speeds are slightly overestimated by VISSIM compared with real speeds. Furthermore, $U$ values are close enough to zero. The closer to zero the coefficient is, the smaller the difference in speeds is. Accordingly, we can conclude that VISSIM is able to well simulate a real traffic situation in terms of microscopic level.

4.3. Preliminary Test. To assess crash risks of all vehicles on the study on-ramp under four different LOS, the concepts of individual risk and societal risk were introduced. The individual risk (IR) is defined as the crash threat to an individual motorist, which is regarded as the likelihood of collision occurring to an individual traveller. The ACI, SCSM, and the difference between TTC and its threshold (if TTC is less than its threshold) all can be viewed as the IR. Building on the notion of IR, the societal risk (SR) index was proposed, which refers to the integrated risk of all individual risks to all of the affected drivers at on-ramp length $L$ measured by surrogate metric $j$, mathematically denoted by

$$
\mathrm{SR}_{j}=\sum_{i=1}^{M} \int_{0}^{L} \operatorname{IR}_{i j}(l) d l \approx \sum_{i=1}^{M} \sum_{l=0}^{N} \operatorname{IR}_{i j}(l) \times i_{\mathrm{dc}},
$$

where $\operatorname{IR}_{i j}(l)$ represents the individual risk of the discrete scenario $i$ at discrete length $l$ measured by surrogate $j, i_{\mathrm{dc}}$ is the interval of two consecutive data collection points, and there are a total of $N$ collection points in length $L$. In this study, 36 data collection points evenly spread on the 360-metre research on-ramp. Due to the complexity of the ACI, the Monte Carlo method (MCM) was used to simplify the calculation of crash probability. 10,000 random drivers' reaction times and MADRs were generated by the MCM in each car following scenario. To make the numerical values more readable, we applied normalization method to process the results of the SCSM. Table 5 presents the SR represented by the TTC, ACI, and SCSM under four varying LOS. As crash data are limited, we cannot conclude which metric has a better ability to predict risks for the study on-ramp through a linear model. However, according to the proportional relation between the SR and crash rates, the performance of the SCSM is optimal, followed by the ACI and TTC. For the TTC, we generally compared the difference between the leading vehicle's speed and the following vehicle's speed to judge a car following scenario safe or risky. This judgement criterion is obviously unreasonable for assessing crash risks for on-ramps, since the speeds of leading vehicles in the merging traffic are larger than those of following vehicles in most cases. However, in a saturated merging traffic stream with smaller time headways and higher driving speeds, even though the abovementioned condition is met, a crash may occur. Accordingly, the traditional TTC is inappropriate for risk assessment for on-ramps. For the ACI, a robust probabilistic causal model enables it to capture any potential car following risks on the on-ramp. However, its complex calculation restricts its applicability in the real world. With the simplified calculation procedure and more or less similar performance to the ACI, the SCSM stand out of these three surrogate measures.

\section{Conclusions and Limitations}

Aiming at rear-end accidents occurring on on-ramps, this paper proposed a new concept of traffic state vulnerability in order to develop and assess the simplified crash surrogate metric (SCSM). As an upgraded version of the traditional time to collision (TTC), the SCSM not only features the same straightforward closed form as the traditional TTC, but also makes up for the shortcoming of the TTC that is unable to accurately assess crash risks in saturated traffic flow. In this study, we used it and another two surrogate measures, aggregated crash index (ACI), and TTC, to predict the crash risks for an on-ramp on Pacific Motorway in 
TABLE 5: Surrogate-based societal risk under four LOS.

\begin{tabular}{|c|c|c|c|c|c|}
\hline \multirow{2}{*}{ LOS } & \multirow{2}{*}{ Crash counts } & \multirow{2}{*}{ Crash rate (counts/hr) } & \multicolumn{3}{|c|}{ SR represented by surrogate metrics } \\
\hline & & & TTC & ACI & SCSM \\
\hline$A \& B$ & 3 & 0.38 & 0 & 119.07 & 29725 \\
\hline $\mathrm{C}$ & 5 & 0.71 & 5.52 & 254.38 & 26540 \\
\hline $\mathrm{D}$ & 8 & 1.33 & 15.91 & 333.67 & 24349 \\
\hline $\mathrm{E}$ & 8 & 2.67 & 19.23 & 397.86 & 21937 \\
\hline
\end{tabular}

Queensland, Australia. Based on a comparative analysis, the TTC based surrogate metric performed the worst, as it makes a concession to limited historical crash counts and boundary condition. Moreover, it is assessed based on the fact that the speeds for leading and following vehicles are invariable during the collision course, which is inconsistent with the real world. When the following vehicle takes necessary evasive actions to avoid a crash, the change in deceleration rates taken by the following vehicle is hard to capture. The SCSM tactfully takes advantage of the concept, the maximum disturbance that a traffic state could accommodate, thereby effectively evading the consideration for the complicated evasive actions involved in a car following scenario. In general, as a variation of the traditional TTC, the SCSM can better assess crash risks in the context of urban environment. The performance of the SCSM is more or less similar to that of the ACI. But considering the ability to resolve practical engineering issues, the SCSM is superior to the ACI. It can assist traffic agents in efficiently and precisely assessing rear-end crash risks for onramps.

The limitations of this research are summarized below. There is no validation for various crash surrogate metrics. Due to the restriction of historical crash counts on the research on-ramp, it is not convincing to only depend on a simple proportional relationship between the SR and crash rates to judge that the SCSM is superior to others. Therefore, we believe that the following solutions can to some extent make up for the limitation. Firstly, the similar on-ramps identified through VISSIM calibration and validation should be grouped together as the study onramps. Secondly, crash counts in each hour occurring on the subject on-ramps need to be extracted from historical crash record. Thirdly, based on the hourly simulation animation for these on-ramps, the results for various surrogate metrics can be obtained. Fourthly, the linear relationships between the SR and crash counts for each surrogate metric can be developed based on 24 points, which will be a sound validation to demonstrate that a certain crash surrogate measure is superior to others. Furthermore, the case study was only focused on an on-ramp on Pacific Motorway. In further study, we will apply these three surrogate measures to assess crash risks for other on-ramps on Pacific Motorway.

\section{Data Availability}

The data used to support the findings of this study are available from the corresponding author upon request.

\section{Conflicts of Interest}

The authors declare no conflicts of interest for publication of this work.

\section{Acknowledgments}

The authors would like to acknowledge the funding provided by the National Natural Science Foundation of China (Grant no. 51378091), the National Natural Science Foundation of Liaoning Province, China (Grant no. 20170540187), and the Fundamental Research Funds for the Central Universities (Grant no. DUT18GJ204).

\section{References}

[1] World Health Organization (WHO), Global Report on Road Safety 2013: Supporting a Decade of Action, WHO Press, World Health Organization, Geneva, Switzerland, 2013.

[2] Department of Infrastructure and Regional Development (DIRD), "Road safety," 2013, http://www.infrastructure.gov.au/ roads/safety.

[3] Z. Liu, Q. Meng, and S. Wang, "Speed-based toll design for cordon-based congestion pricing scheme," Transportation Research Part C: Emerging Technologies, vol. 31, pp. 83-98, 2013.

[4] Z. Liu, S. Wang, and Q. Meng, "Optimal joint distance and time toll for cordon-based congestion pricing," Transportation Research Part B: Methodological, vol. 69, pp. 81-97, 2014.

[5] M. Zhou, X. Qu, and X. Li, "A recurrent neural network based microscopic car following model to predict traffic oscillation," Transportation Research Part C: Emerging Technologies, vol. 84, pp. 245-264, 2017.

[6] United States Department of Transportation (US DOT), Traffic Safety Fact 2005, NHTSA's national center for statistic and analysis, 2005, http://www-nrd.nhtsa.dot.gov/Pubs/810631.pdf.

[7] L. Zhang, C. Chen, J. Zhang, S. Fang, J. You, and J. Guo, "Modeling Lane-Changing Behavior in Freeway Off-Ramp Areas from the Shanghai Naturalistic Driving Study," Journal of Advanced Transportation, vol. 2018, pp. 1-10, 2018.

[8] X. Qu, S. Wang, and J. Zhang, "On the fundamental diagram for freeway traffic: a novel calibration approach for single-regime models," Transportation Research Part B: Methodological, vol. 73, pp. 91-102, 2015.

[9] X. Qu, J. Zhang, and S. Wang, "On the stochastic fundamental diagram for freeway traffic: Model development, analytical properties, validation, and extensive applications," Transportation Research Part B: Methodological, vol. 104, pp. 256-271, 2017.

[10] H. Wu, L. Gao, and Z. Zhang, "Analysis of crash data using quantile regression for counts," Journal of Transportation Engineering, vol. 140, no. 4, Article ID 04013025, 2014. 
[11] C. Xu, H. Li, J. Zhao, J. Chen, and W. Wang, "Investigating the relationship between jobs-housing balance and traffic safety," Accident Analysis \& Prevention, vol. 107, pp. 126-136, 2017.

[12] C. Xu, Y. Wang, P. Liu, W. Wang, and J. Bao, "Quantitative risk assessment of freeway crash casualty using high-resolution traffic data," Reliability Engineering \& System Safety, vol. 169, pp. 299-311, 2018.

[13] D. Lord, S. D. Guikema, and S. R. Geedipally, "Application of the Conway-Maxwell-Poisson generalized linear model for analyzing motor vehicle crashes," Accident Analysis \& Prevention, vol. 40, no. 3, pp. 1123-1134, 2008.

[14] S. Jin, X. Qu, C. Xu, D. Ma, and D. Wang, "An improved multivalue cellular automata model for heterogeneous bicycle traffic flow," Physics Letters A, vol. 379, no. 39, Article ID 23345, pp. 2409-2416, 2015.

[15] H.-C. Chin and S.-T. Quek, "Measurement of traffic conflicts," Safety Science, vol. 26, no. 3, pp. 169-185, 1997.

[16] D. Gettman and L. Head, "Surrogate safety measures from traffic simulation models," Transportation Research Record, no. 1840, pp. 104-115, 2003.

[17] A. P. Tarko, "Use of crash surrogates and exceedance statistics to estimate road safety," Accident Analysis \& Prevention, vol. 45, pp. 230-240, 2012.

[18] C. Chai, X. Shi, and Y. D. Wong, "Estimating Safety Effects of Green-Man Countdown Devices at Signalized Pedestrian Crosswalk Based on Cellular Automata," Journal of Advanced Transportation, vol. 2017, pp. 1-13, 2017.

[19] T. D. Chu, T. Miwa, and T. Morikawa, "Discrete choice models for gap acceptance at urban expressway merge sections considering safety, road geometry, and traffic conditions," Journal of Transportation Engineering, vol. 143, no. 7, Article ID 04017025, 2017.

[20] E. Hauer, "Traffic conflicts and exposure," Accident Analysis \& Prevention, vol. 14, no. 5, pp. 359-364, 1982.

[21] A. Tarko, G. Davis, N. Saunier, T. Sayed, and S. Washington, White paper: Surrogate Measures of Safety, Committee on Safety Data Evaluation and Analysis (ANB20), 2009.

[22] Y. Kuang, X. Qu, and S. Wang, "A tree-structured crash surrogate measure for freeways," Accident Analysis \& Prevention, vol. 77, pp. 137-148, 2015.

[23] J. Hayward, "Near miss determination through use of a scale of danger: Transportation Research Record," Journal of the Transportation Research Board, vol. 384, pp. 24-34, 1972.

[24] R. Van der Horst, Time-to-collision as a cue for decision-making in braking, North-Holland, Amsterdam, 1991.

[25] F. F. Saccomanno, F. Cunto, G. Guido, and A. Vitale, "Comparing safety at signalized intersections and roundabouts using simulated rear-end conflicts," Transportation Research Record, vol. 2078, pp. 90-95, 2008.

[26] Q. Meng and X. Qu, "Estimation of vehicle crash frequencies in road tunnels," Accident Analysis \& Prevention, vol. 48, pp. 254263, 2012.

[27] J. Weng, J.-Z. Zhu, X. Yan, and Z. Liu, "Investigation of work zone crash casualty patterns using association rules," Accident Analysis \& Prevention, vol. 92, pp. 43-52, 2016.

[28] X. Qu, Y. Kuang, E. Oh, and S. Jin, "Safety evaluation for expressways: a comparative study for macroscopic and microscopic indicators," Traffic Injury Prevention, vol. 15, no. 1, pp. 89-93, 2013.

[29] X. Qu, Y. Yang, Z. Liu, S. Jin, and J. Weng, "Potential crash risks of expressway on-ramps and off-ramps: a case study in Beijing, China," Safety Science, vol. 70, pp. 58-62, 2014.
[30] R. Fan, H. Yu, P. Liu, and W. Wang, "Using VISSIM simulation model and surrogate safety assessment model for estimating field measured traffic conflicts at freeway merge areas," IET Intelligent Transport Systems, vol. 7, no. 1, pp. 68-77, 2013. 


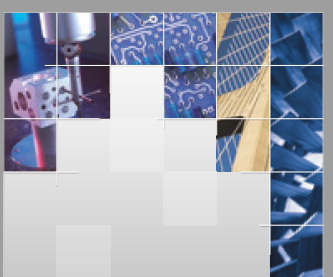

\section{Enfincering}
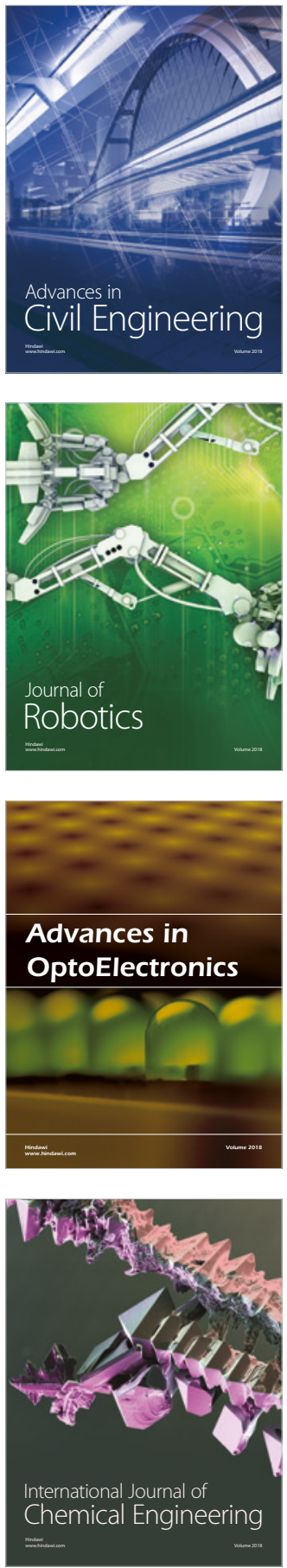

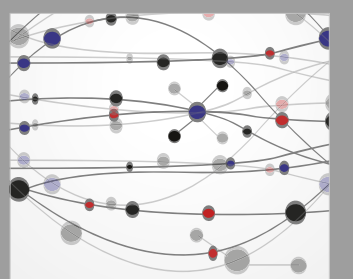

\section{Rotating \\ Machinery}

The Scientific World Journal

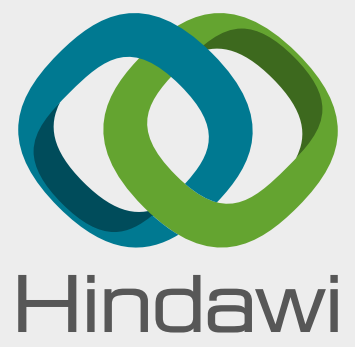

Submit your manuscripts at

www.hindawi.com
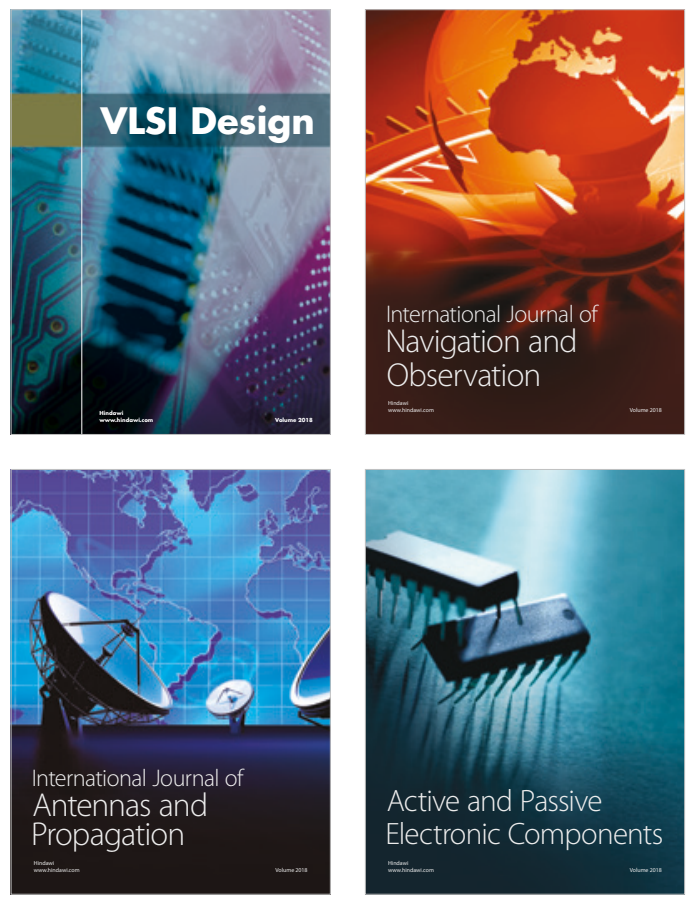
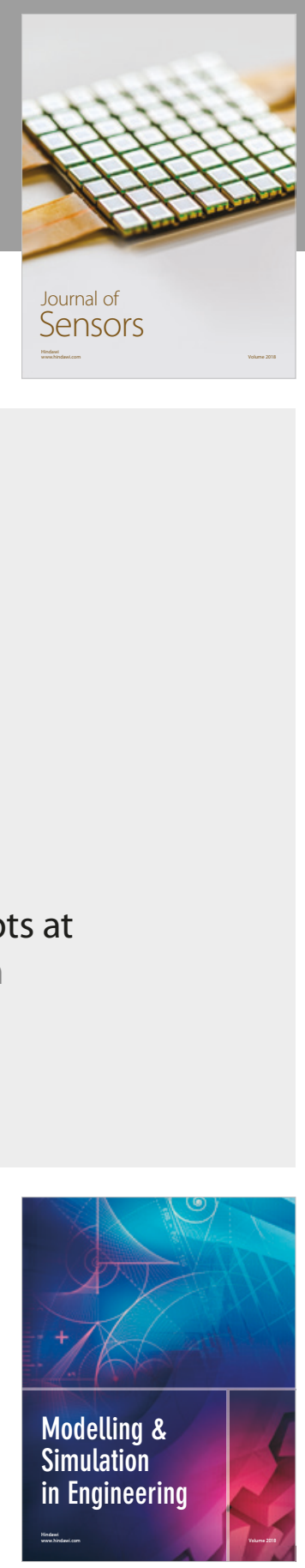

\section{Advances \\ Multimedia}
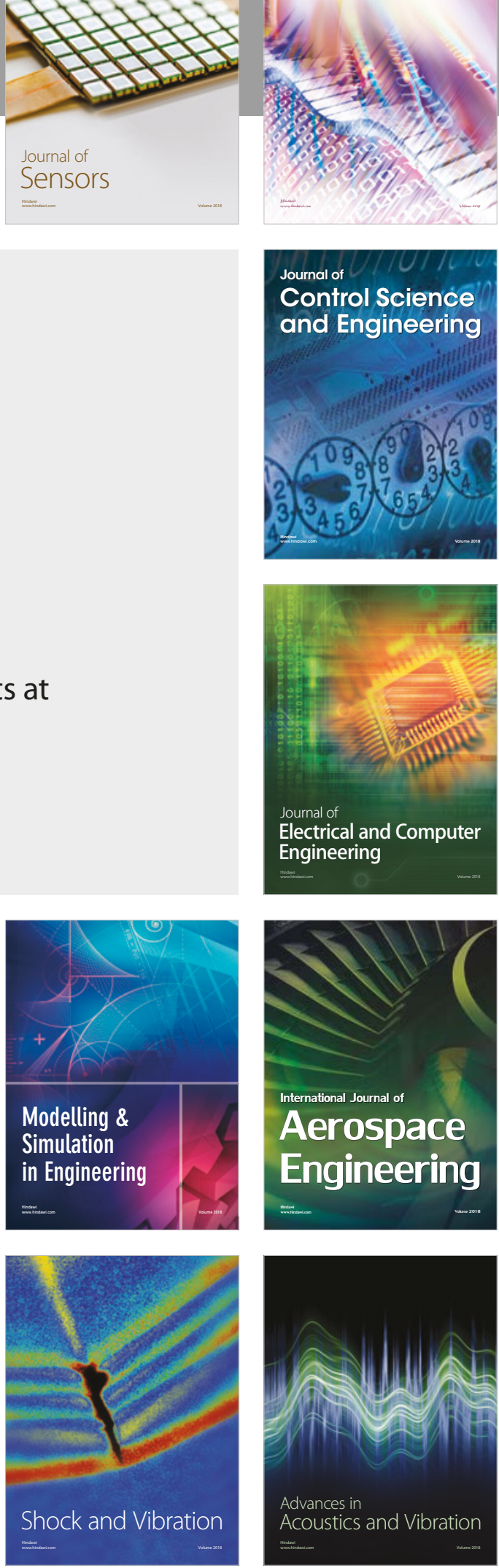